\title{
Imaginary quadratic fields satisfying the Hilbert-Speiser type condition for a small prime $p$
}

\author{
by \\ Humio ICHIMURA (Ibaraki) \\ and Hiroki Sumida-TAKahashi (Tokushima)
}

1. Introduction. Let $p$ be a prime number, and $\Gamma=\Gamma_{p}$ the cyclic group of order $p ; \Gamma=\mathbb{F}_{p}^{+}$, where $\mathbb{F}_{p}^{+}$is the additive group of the finite field $\mathbb{F}_{p}$ of $p$ elements. We say that a number field $F$ satisfies condition $\left(A_{p}\right)$ if for any tame $\Gamma$-extension $N / F, \mathcal{O}_{N}$ is cyclic over the group $\operatorname{ring} \mathcal{O}_{F} \Gamma$. Here, $\mathcal{O}_{F}$ is the ring of integers of $F$. It is well known by work of Hilbert and Speiser that the rationals $\mathbb{Q}$ satisfy $\left(A_{p}\right)$ for all primes $p$. In $[6$, Theorem 1], Greither et $a l$. gave a necessary condition for a number field $F$ to satisfy $\left(A_{p}\right)$ in terms of (a subgroup of) the ray class group of $F$ defined modulo $p$, using a theorem of McCulloh $[20,21]$. Applying that condition, they proved that $F \neq \mathbb{Q}$ does not satisfy $\left(A_{p}\right)$ for infinitely many primes $p$ ([6, Theorem 2$\left.]\right)$. Thus, it is of interest to determine which number fields $F$ satisfy $\left(A_{p}\right)$. Several authors $[3,4,11-13]$ obtained some results on the problem using the above mentioned condition (and some other results such as a theorem of Gómez Ayala [5, Theorem 2.1]). For instance, it was shown by Carter [3, Corollary 3] that an imaginary quadratic field $F=\mathbb{Q}(\sqrt{-d})$ with $d>0$ square free satisfies $\left(A_{2}\right)$ if and only if $d=1,3$ or 7 . Further, all quadratic fields satisfying $\left(A_{3}\right)$ were determined independently in [3, Corollary 5] and [12, Proposition]. There are exactly four imaginary and eight real ones satisfying $\left(A_{3}\right)$. The purpose of this paper is to determine all imaginary quadratic fields satisfying $\left(A_{p}\right)$ for $p=5,7$ or 11 . The result is as follows:

THEOREM 1. An imaginary quadratic field $F=\mathbb{Q}(\sqrt{-d})$ with a square free positive integer $d$ satisfies the condition $\left(A_{5}\right)$ if and only if $d=1$ or 3 . It satisfies $\left(A_{7}\right)$ if and only if $d=3$. No imaginary quadratic field satisfies $\left(A_{11}\right)$.

As in [6], the above mentioned theorem of McCulloh plays an important role in proving Theorem 1. In Section 2, we recall McCulloh's theorem and

2000 Mathematics Subject Classification: 11R33, 11R11. 
several of its consequences including the above mentioned condition for $\left(A_{p}\right)$ in [6]. In Section 3, we give some conditions for an imaginary quadratic field to satisfy $\left(A_{p}\right)$ and prove Theorem 1 . In Section 4 , we review some topics on subfields of the $p$-cyclotomic field $\mathbb{Q}\left(\zeta_{p}\right)$ satisfying $\left(A_{p}\right)$.

2. Consequences of McCulloh's theorem. In this section, we recall a theorem of McCulloh $[20,21]$ and several of its consequences. Let $F$ be a number field. For an integer $a \in \mathcal{O}_{F}$, let $C l_{F}(a)$ be the ray class group of $F$ defined modulo the ideal $a \mathcal{O}_{F}$. We simply write $C l_{F}=C l_{F}(1)$, the absolute class group of $F$. Let $C l\left(\mathcal{O}_{F} \Gamma\right)$ be the locally free class group of the group ring $\mathcal{O}_{F} \Gamma$, and let $C l^{0}\left(\mathcal{O}_{F} \Gamma\right)$ be the kernel of the homomorphism $C l\left(\mathcal{O}_{F} \Gamma\right) \rightarrow C l_{F}$ induced from the augmentation $\mathcal{O}_{F} \Gamma \rightarrow \mathcal{O}_{F}$. The class group $C l^{0}\left(\mathcal{O}_{F} \Gamma\right)$ is known to be a quotient of some copies of the ray class group $C l_{F\left(\zeta_{p}\right)}(p)$, but it is a quite complicated object in general. Let $R\left(\mathcal{O}_{F} \Gamma\right)$ be the subset of $\operatorname{Cl}\left(\mathcal{O}_{F} \Gamma\right)$ consisting of the locally free classes $\left[\mathcal{O}_{N}\right]$ for all tame $\Gamma$-extensions $N / F$. It follows that $F$ satisfies $\left(A_{p}\right)$ if and only if $R\left(\mathcal{O}_{F} \Gamma\right)=\{0\}$. It is known that $R\left(\mathcal{O}_{F} \Gamma\right) \subseteq C l^{0}\left(\mathcal{O}_{F} \Gamma\right)$. Let $G=\mathbb{F}_{p}^{\times}$be the multiplicative group of $\mathbb{F}_{p}$. Through the natural action of $G$ on $\Gamma=\mathbb{F}_{p}^{+}$, the group ring $\mathbb{Z} G$ acts on $C l\left(\mathcal{O}_{F} \Gamma\right)$. Let $\mathcal{S}_{G}$ be the classical Stickelberger ideal of the group ring $\mathbb{Z} G$. For the definition, see Washington [26, Chapter 6].

TheOREM 2 ([21]). Under the above setting, we have

$$
R\left(\mathcal{O}_{F} \Gamma\right)=C l^{0}\left(\mathcal{O}_{F} \Gamma\right)^{\mathcal{S}_{G}} .
$$

Let $\mathcal{O}_{F}^{\times}$be the group of units of a number field $F$. For an integer $a \in \mathcal{O}_{F}$, let $\left[\mathcal{O}_{F}^{\times}\right]_{a}$ be the subgroup of the multiplicative group $\left(\mathcal{O}_{F} / a\right)^{\times}$consisting of the classes containing a unit of $F$. The quotient $\left(\mathcal{O}_{F} / a\right)^{\times} /\left[\mathcal{O}_{F}^{\times}\right]_{a}$ is a subgroup of the ray class group $C l_{F}(a)$. Greither et al. [6] proved the following relation between condition $\left(A_{p}\right)$ and $C l_{F}(p)$ from Theorem 2 by studying a canonical subgroup of $\mathrm{Cl}\left(\mathcal{O}_{F} \Gamma\right)$, called the Swan subgroup.

Proposition 1 ([6, Theorem 1]). Assume that a number field $F$ satisfies condition $\left(A_{p}\right)$. Then the exponent of the quotient $\left(\mathcal{O}_{F} / p\right)^{\times} /\left[\mathcal{O}_{F}^{\times}\right]_{p}$ divides $(p-1)^{2} / 2$ when $p \geq 3$, and $\left(\mathcal{O}_{F} / p\right)^{\times}=\left[\mathcal{O}_{F}^{\times}\right]_{p}$ when $p=2$.

The following is obtained from Proposition 1 and [5, Theorem 2.1].

Proposition 2 ([11, Proposition 2]). A number field $F$ satisfies condition $\left(A_{2}\right)$ if and only if the ray class group $C l_{F}(2)$ is trivial.

Similar conditions for $\left(A_{2}\right)$ are also given in [3, Theorem 2] and in Herreng [9, Theorem 2.1]. In view of Proposition 2, we let $p \geq 3$ in the following. To give another consequence of Theorem 2, we need to recall a "Stickelberger ideal" associated to a subgroup of $G$. Let $H$ be a subgroup of $G$. For an el- 
ement $\alpha \in \mathbb{Z} G$, let

$$
\alpha_{H}=\sum_{\sigma \in H} a_{\sigma} \sigma \in \mathbb{Z} H \quad \text { with } \quad \alpha=\sum_{\sigma \in G} a_{\sigma} \sigma .
$$

In other words, $\alpha_{H}$ is the $H$-part of $\alpha$. In [14], we defined the Stickelberger ideal $\mathcal{S}_{H}$ of $\mathbb{Z} H$ by

$$
\mathcal{S}_{H}=\left\{\alpha_{H} \mid \alpha \in \mathcal{S}_{G}\right\} \subseteq \mathbb{Z} H
$$

Several properties of the ideal $\mathcal{S}_{H}$ are studied in $[14,15,17,18]$. For an integer $i \in \mathbb{Z}$, let $\bar{i}$ be the class in $\mathbb{F}_{p}=\mathbb{Z} / p$ containing $i$. It is known that the ideal $\mathcal{S}_{H}$ is generated over $\mathbb{Z}$ by the Stickelberger elements

$$
\theta_{H, r}=\sum_{i}^{\prime}\left[\frac{r i}{p}\right] \cdot \bar{i}^{-1} \in \mathbb{Z} H
$$

for all integers $r \in \mathbb{Z}$. Here, $i$ runs over the integers with $1 \leq i \leq p-1$ and $\bar{i} \in H$, and for a real number $x,[x]$ is the largest integer $\leq x$. Let $N_{H}$ be the norm element of $\mathbb{Z} H$. It follows that

$$
N_{H}=-\theta_{H,-1} \in \mathcal{S}_{H} \text {. }
$$

Letting $\varrho$ be a generator of $H$, put

$$
\mathfrak{n}_{H}= \begin{cases}1+\varrho+\cdots+\varrho^{|H| / 2-1} & \text { if }|H| \text { is even } \\ 1 & \text { if }|H| \text { is odd }\end{cases}
$$

As is easily seen, the ideal $\left\langle\mathfrak{n}_{H}\right\rangle=\mathfrak{n}_{H} \mathbb{Z} H$ does not depend on the choice of $\varrho$. It is known that $\mathcal{S}_{H} \subseteq\left\langle\mathfrak{n}_{H}\right\rangle\left(\left[18\right.\right.$, Lemma 1]) and that the quotient $\left\langle\mathfrak{n}_{H}\right\rangle / \mathcal{S}_{H}$ is a finite abelian group whose order divides the relative class number $h_{p}^{-}$of the $p$-cyclotomic field $\mathbb{Q}\left(\zeta_{p}\right)([18$, Theorem 2$])$ :

$$
\left[\left\langle\mathfrak{n}_{H}\right\rangle: \mathcal{S}_{H}\right] \mid h_{p}^{-} \text {. }
$$

Let $F$ be a number field, and $K=F\left(\zeta_{p}\right)$. We naturally identify the Galois group $\operatorname{Gal}(K / F)$ with a subgroup $H$ of $G$ through the Galois action on $\zeta_{p}$. Then the group ring $\mathbb{Z} H$ acts on several objects associated to $K / F$. Let $\pi=\zeta_{p}-1$. The following assertion was obtained from Theorem 2 and Proposition 1.

Proposition 3 ([13, Theorem 5]). Let $F$ be a number field, and let $K=F\left(\zeta_{p}\right)$ and $H=\operatorname{Gal}(K / F) \subseteq G$. If $F$ satisfies $\left(A_{p}\right)$, then

$$
C l_{K}(\pi)^{\mathcal{S}_{H}}=\{0\} \quad \text { and } \quad C l_{K}(p)^{\mathcal{S}_{H}} \cap C l_{K}(p)^{H}=\{0\} .
$$

Here, $C l_{K}(p)^{H}$ is the Galois invariant part.

It is known that the converse of this assertion holds when $p=3$ ([12, Theorem 2]). The following is a consequence of Proposition 3.

Proposition 4. Let $F$ and $K$ be as in Proposition 3. Assume that $F$ satisfies $\left(A_{p}\right)$ and that the norm map $C l_{K} \rightarrow C l_{F}$ is surjective. Then 
the natural map $C l_{F} \rightarrow C l_{K}$ is trivial. In particular, the exponent of $C l_{F}$ divides $[K: F]$.

Proof. By the assumption, any ideal class $c \in C l_{F}$ is of the form $c=d^{N_{H}}$ for some $d \in C l_{K}$. However, when $F$ satisfies $\left(A_{p}\right)$, the class $d^{N_{H}}$ is trivial in $C l_{K}$ by Proposition 3 and $N_{H} \in \mathcal{S}_{H}$.

When $F / \mathbb{Q}$ is unramified at $p$, the Galois group $\operatorname{Gal}(K / F)$ is naturally identified with $G=\mathbb{F}_{p}^{\times}$through the Galois action on $\zeta_{p}$. The following is a consequence of Theorem 2.

Proposition 5. Assume that $F / \mathbb{Q}$ is unramified at $p$, and let $K=$ $F\left(\zeta_{p}\right)$. Then $F$ satisfies condition $\left(A_{p}\right)$ if and only if the Stickelberger ideal $\mathcal{S}_{G}$ annihilates the ray class group $C l_{K}(\pi)$.

Proof. Brinkhuis [2, Proposition (2.2)] proved that the $\mathbb{Z} G$-module $C l^{0}\left(\mathcal{O}_{F} \Gamma\right)$ is naturally isomorphic to the ray class group $C l_{K}(\pi)$ when $F / \mathbb{Q}$ is unramified at $p$. Hence, the assertion follows immediately from Theorem 2.

Though the following assertion is irrelevant to the proof of Theorem 1 , it might be of some interest to the reader. For a CM-field $K$, let $C l_{K}^{-}$be the kernel of the norm map $C l_{K} \rightarrow C l_{K^{+}}$where $K^{+}$is the maximal real subfield of $K$.

Proposition 6. Let $F$ be a totally real number field, and $K=F\left(\zeta_{p}\right)$. If $F$ satisfies $\left(A_{p}\right)$, then the exponent of $C l_{K}^{-}$divides $2 h_{p}^{-}$.

Proof. Let $H=\operatorname{Gal}(K / F) \subseteq G$, and let $\varrho$ be a generator of $H$. As $F$ is totally real, $|H|$ is even and $J=\varrho^{|H| / 2}$ is the complex conjugation in $H$. We easily see that $(1-\varrho) \mathfrak{n}_{H}=1-J$, and that $\mathfrak{n}_{H} h_{p}^{-} \in \mathcal{S}_{H}$ by $(2)$. Hence, $(1-J) h_{p}^{-} \in \mathcal{S}_{H}$. Assume that $F$ satisfies $\left(A_{p}\right)$. Then, by Proposition 3, $(1-J) h_{p}^{-}$annihilates $C l_{K}$. The assertion follows from this.

3. Imaginary quadratic fields. In this section, let $p \geq 3$ be an odd prime number, and $F=\mathbb{Q}(\sqrt{-d})$ an imaginary quadratic field with a square free positive integer $d$.

Lemma 1 . When $p$ is ramified in $F / \mathbb{Q}, F$ satisfies $\left(A_{p}\right)$ if and only if $p=3$ and $F=\mathbb{Q}(\sqrt{-3})$.

Proof. The "only if" part is an easy consequence of Proposition 1 since $\left(\mathcal{O}_{F} / p\right)^{\times}$is cyclic of order $p(p-1)$ when $p$ ramifies in $F$. The "if" part is due to $[5$, p. 110].

Lemma 2.

(I) Let $p=3$ or 5 . If $F \neq \mathbb{Q}(\sqrt{-1}), \mathbb{Q}(\sqrt{-3})$ and $p$ is inert in $F$, then $F$ does not satisfy $\left(A_{p}\right)$. 
(II) Let $p \geq 7$. If $p$ is inert in $F$, then $F$ does not satisfy $\left(A_{p}\right)$.

Proof. This is an easy consequence of Proposition 1 since $\left(\mathcal{O}_{F} / p\right)^{\times}$is cyclic of order $p^{2}-1$ when $p$ is inert in $F$.

In all what follows, we exclude the case where $p=3$ and $F=\mathbb{Q}(\sqrt{-3})$, and we let $K=F\left(\zeta_{p}\right)$. Hence, by Lemma 1 , if $F$ satisfies $\left(A_{p}\right)$, then $F / \mathbb{Q}$ is unramified at $p$ and the Galois group $\operatorname{Gal}(K / F)$ is naturally identified with $G=\mathbb{F}_{p}^{\times}$.

LEMmA 3. If $F$ satisfies $\left(A_{p}\right)$, then the exponent of the class group $C l_{F}$ divides 2.

Proof. We use a standard argument in [26, pp. 289-290]. Assume that $F$ satisfies $\left(A_{p}\right)$. As $F / \mathbb{Q}$ is unramified at $p, K / F$ is totally ramified at the primes over $p$. Hence, the natural map $C l_{F} \rightarrow C l_{K}$ is trivial by Proposition 4 . Let $\mathfrak{A}$ be an arbitrary ideal of $F$ relatively prime to $p$. We have $\mathfrak{A O}_{K}=\alpha \mathcal{O}_{K}$ for some $\alpha \in K^{\times}$. Let $\varrho$ be a generator of $G$, and $J$ a generator of $\operatorname{Gal}(F / \mathbb{Q})=\operatorname{Gal}\left(K / K^{+}\right)$where $K^{+}$is the maximal real subfield of $K$. As $\mathfrak{A}$ is an ideal of $F$, we have $\alpha^{1-\varrho}=\varepsilon \in \mathcal{O}_{K}^{\times}$. On the other hand, $\mathfrak{A}^{1+J}=\beta \mathcal{O}_{F}$ for some $\beta \in \mathbb{Q}^{\times}$. Hence, $\alpha^{1+J}=\beta \eta$ for some unit $\eta \in \mathcal{O}_{K}^{\times}$. It follows that

$$
\varepsilon^{1+J}=\left(\alpha^{1+J}\right)^{1-\varrho}=\eta^{1-\varrho}
$$

as $\beta \in \mathbb{Q}^{\times}$. Putting $\alpha_{1}=\alpha^{2} / \eta$, we have

$$
\alpha_{1} \mathcal{O}_{K}=\mathfrak{A}^{2} \mathcal{O}_{K}
$$

Let

$$
\varepsilon_{1}=\alpha_{1}^{\varrho-1}=\varepsilon^{-2} \eta^{1-\varrho} \in \mathcal{O}_{K}^{\times}
$$

Then

$$
\varepsilon_{1}^{1+J}=\varepsilon^{-2(1+J)} \eta^{(1-\varrho)(1+J)}=\eta^{(1-J)(\varrho-1)} .
$$

Hence, $\varepsilon_{1}$ is a root of unity in $K$ by a theorem on units of a CM-field (cf. [26, Theorem 4.12]). Let $\mu_{p}$ be the group of $p$ th roots of unity in $K$. We consider separately the cases when $\varepsilon_{1} \in \mu_{p}$ or not.

The case $\varepsilon_{1} \in \mu_{p}$. Since the map $\varrho-1: \mu_{p} \rightarrow \mu_{p}$ is an isomorphism, we can write $\varepsilon_{1}=\zeta^{\varrho-1}$ for some $\zeta \in \mu_{p}$. Hence, it follows from (4) that $\left(\alpha_{1} / \zeta\right)^{\varrho}=\alpha_{1} / \zeta$ and $\alpha_{1} / \zeta \in F^{\times}$. Therefore, by $(3), \mathfrak{A}^{2}$ is a principal ideal of $F$.

The case $\varepsilon_{1} \notin \mu_{p}$. As the class groups of $\mathbb{Q}(\sqrt{-1})$ and $\mathbb{Q}(\sqrt{-3})$ are trivial, we may well assume that $F \neq \mathbb{Q}(\sqrt{-1}), \mathbb{Q}(\sqrt{-3})$. Then the condition $\varepsilon_{1} \notin \mu_{p}$ implies that $-\varepsilon_{1} \in \mu_{p}$, and hence, $-\varepsilon_{1}=\zeta^{\varrho-1}$ for some $\zeta \in \mu_{p}$. On the other hand, we have $-1=\left(\sqrt{p^{*}}\right)^{\varrho-1}$ where $p^{*}=p$ if $p \equiv 1 \bmod 4$ and $p^{*}=-p$ otherwise. Therefore, $\varepsilon_{1}=\left(\sqrt{p^{*} \zeta}\right)^{\varrho-1}$. Hence, it follows from $(4)$ 
that

$$
\left(\alpha_{1} / \sqrt{p^{*}} \zeta\right)^{\varrho}=\alpha_{1} / \sqrt{p^{*}} \zeta \text { and } \alpha_{1} / \sqrt{p^{*}} \zeta \in F^{\times} .
$$

This implies that $p$ is ramified in $F$ as $\mathfrak{A}$ is relatively prime to $p$. This is a contradiction.

Lemma 3 asserts that if the exponent of $C l_{F}$ is greater than 2 , then $F$ does not satisfy $\left(A_{p}\right)$ for any prime $p$. All imaginary quadratic fields $F$ with $C l_{F}^{2}=\{0\}$ were determined by Weinberger [27, Theorem 1] with possibly one exception. A table of such $F$ 's is given in Miyada [22, p. 539]. There are exactly 65 (or possibly 66) such $F$. In particular, we obtain the following:

Proposition 7. For each prime number $p$, there exist at most 65 (or possibly 66) imaginary quadratic fields satisfying condition $\left(A_{p}\right)$.

Lemma 4. Let $p=5$, and $E=F(\sqrt{5})$. If $F$ satisfies $\left(A_{5}\right)$, then the natural map $C l_{F} \rightarrow C l_{E}$ is trivial.

Proof. Assume that $F$ satisfies $\left(A_{5}\right)$. Let $\varrho$ be a generator of $G=$ $\operatorname{Gal}(K / F)$. We have $\mathcal{S}_{G}=\langle 1+\varrho\rangle$ by $h_{5}^{-}=1$ and $(2)$. By the assumption and Proposition 3 or $5, c^{1+\varrho}=1$ for any $c \in C l_{K}$. As the norm map $C l_{K} \rightarrow C l_{E}$ is surjective, this relation holds for any $c \in C l_{E}$. As the norm map $C l_{E} \rightarrow C l_{F}$ is surjective, any class $d \in C l_{F}$ is of the form $d=N_{E / F}(c)=c^{1+\varrho}$ for some $c \in C l_{E}$. Therefore, we obtain the assertion.

Lemma 5. Let $p$ be a prime number with $p \equiv 3 \bmod 4$, and $E=F(\sqrt{-p})$. If $F$ satisfies $\left(A_{p}\right)$, then the natural map $C l_{F} \rightarrow C l_{E}$ is trivial.

Proof. Assume that $F$ satisfies $\left(A_{p}\right)$. Let $\mathfrak{A}$ be an ideal of $F$. By Proposition $4, \mathfrak{A O}_{K}=\alpha \mathcal{O}_{K}$ for some $\alpha \in K^{\times}$. Hence, $\mathfrak{A}^{[K: E]} \mathcal{O}_{E}=\beta \mathcal{O}_{E}$ with $\beta=N_{K / E}(\alpha)$. This implies that $\mathfrak{A \mathcal { O } _ { E }}$ is a principal ideal since $[K: E]$ is odd by the assumption on $p$, and $\mathfrak{A}^{2}$ is principal in $F$ by Lemma 3 .

Lemma 6. Let $p$ be a prime number with $p \equiv 3 \bmod 4$ or $p=5$. If $F$ satisfies $\left(A_{p}\right)$, then $C l_{F}$ is isomorphic to the abelian group $(\mathbb{Z} / 2)^{\oplus R}$ with $R \leq 2$.

Proof. Let $H_{F}^{(2)} / F$ be the maximal unramified abelian extension of exponent 2, and let $E$ be as in Lemmas 4 and 5 . Assume that $F$ satisfies $\left(A_{p}\right)$. Then $\left[H_{F}^{(2)}: F\right]=\left[H_{F}^{(2)} E: E\right]$ since $E / F$ is totally ramified at the primes over $p$. Let $t$ be the number of prime numbers which ramify in $F$. Let $\lambda_{1}, \ldots, \lambda_{r}$ (resp. $\mu_{1}, \ldots, \mu_{s}$ ) be all the odd prime numbers which ramify in $F$ and are congruent to 1 (resp. 3) modulo 4 . The 2-rank of $C l_{F}$ equals $t-1$ by a well known theorem on quadratic fields (cf. Hecke [8, Theorem 132]). Hence, by Lemma 3 , it suffices to show that $t \leq 3$ since we are assuming that $F$ satisfies $\left(A_{p}\right)$. It is well known and easy to show that

$$
H_{F}^{(2)}=F\left(\sqrt{\lambda_{i}}, \sqrt{-\mu_{j}} \mid 1 \leq i \leq r, 1 \leq j \leq s\right) .
$$


Let $\ell$ be any one of the prime numbers $\lambda_{i}$ and $\mu_{j}$, and let $\mathfrak{L}$ be the prime ideal of $F$ over $\ell$. By Lemmas 4 and 5, the ideal $\mathfrak{L O}_{E}$ is principal. This implies that $\ell=\varepsilon x^{2}$ for some unit $\varepsilon \in \mathcal{O}_{E}^{\times}$and $x \in E^{\times}$. Therefore,

$$
H_{F}^{(2)} E \subseteq E\left(\sqrt{\varepsilon} \mid \varepsilon \in \mathcal{O}_{E}^{\times}\right) .
$$

Now, from the above, it follows that

$$
2^{t-1}=\left[H_{F}^{(2)}: F\right]=\left[H_{F}^{(2)} E: E\right]=1,2 \text { or } 4
$$

since the group $\mathcal{O}_{E}^{\times}$is generated by two elements. Therefore, $t \leq 3$.

For a number field $N$ and a prime number $q$, let $C l_{N}[q]$ be the Sylow $q$-subgroup of the class group $C l_{N}$.

Lemma 7. Let $p \geq 7$ be a prime number with $p \equiv 3 \bmod 4$. Let $K=F\left(\zeta_{p}\right)$, and let $N$ be an intermediate field of $K / F$ with $2 \nmid[K: N]$. If the 2-part $C l_{N}[2]$ is nontrivial and cyclic as an abelian group, then $F$ does not satisfy $\left(A_{p}\right)$.

Proof. Assume that $C l_{N}[2]$ is nontrivial and cyclic, but $F$ satisfies $\left(A_{p}\right)$. Let $c$ be a generator of the cyclic group $C l_{N}[2]$. Then

$$
c^{\sigma} \equiv c \bmod 2 C l_{N}[2]
$$

for all $\sigma \in G$. As $[K: N]$ is odd, the natural map $C l_{N}[2] \rightarrow C l_{K}$ is injective. Let $\bar{c}$ and $\overline{C l}_{N}[2]$ be the images of $c$ and $C l_{N}[2]$ under this injection. As $F$ satisfies $\left(A_{p}\right)$, the Stickelberger element $\theta_{G, 2}$ kills $\bar{c}$. We easily see that the augmentation $\mathbb{Z} G \rightarrow \mathbb{Z}$ maps the element $\theta_{G, 2}$ to $(p-1) / 2$ from the definition (1). Therefore, it follows from (5) that

$$
1=\bar{c}^{\theta_{G, 2}} \equiv \bar{c}^{(p-1) / 2} \bmod 2 \overline{C l}_{N}[2] .
$$

This implies that $c^{(p-1) / 2} \in 2 C l_{N}[2]$ as $C l_{N}[2] \rightarrow C l_{K}$ is injective. Hence, $c \in 2 C l_{N}[2]$ as $(p-1) / 2$ is odd. This is a contradiction.

For a number field $N$, let $h_{N}$ be the class number of $N$.

LEMMA 8. Let $p$ be a prime number with $p \equiv 3 \bmod 4$ and $p \leq 19$, and let $E=F(\sqrt{-p})$. If the class number $h_{E}$ is divisible by an odd prime number $q$ relatively prime to $(p-1) / 2$, then $F$ does not satisfy $\left(A_{p}\right)$.

Proof. As $q$ is relatively prime to $(p-1) / 2$, the natural map $C l_{E}[q] \rightarrow C l_{K}$ is injective. Let $c$ be a class in $C l_{E}$ of order $q$, and $\bar{c}$ its lift to $K$. The class $\bar{c}$ is nontrivial. Let $\varrho$ be a generator of $G=\operatorname{Gal}(K / F)$. Assume that $F$ satisfies $\left(A_{p}\right)$. Then $c^{\varrho}=c^{-1}$ since $h_{F}$ is a power of 2 by Lemma 3 . Hence,

$$
\bar{c}^{\varrho}=\bar{c}^{-1} \text {. }
$$


The condition $p \leq 19$ is equivalent to $h_{p}^{-}=1$ (cf. [26, Corollary 11.18]). Hence, by (2), the Stickelberger ideal $\mathcal{S}_{G}$ is generated by $\mathfrak{n}_{G}$. Since $F$ satisfies $\left(A_{p}\right)$, we see that $\mathfrak{n}_{G}$ annihilates $C l_{K}$ by Proposition 3 or 5 . As $(p-1) / 2$ is odd, we see from (6) that

$$
1=\bar{c}^{\mathfrak{n}_{G}}=\bar{c}^{\{1+(-1)\}+\cdots+\{1+(-1)\}+1}=\bar{c} .
$$

This is a contradiction.

Lemma 9. Let $F$ be a quadratic field not necessarily imaginary, and let $p$ be a prime number splitting in $F$. Let $\mathfrak{P}_{1}$ and $\mathfrak{P}_{2}$ be the prime ideals of $K=$ $F\left(\zeta_{p}\right)$ over $p$. Then the Stickelberger ideal $\mathcal{S}_{G}$ annihilates $\left(\mathcal{O}_{K} / \pi\right)^{\times} /\left[\mathcal{O}_{K}^{\times}\right]_{\pi}$ if and only if there exists a unit $\varepsilon \in \mathcal{O}_{K}^{\times}$satisfying

$$
\varepsilon \equiv 1 \bmod \mathfrak{P}_{1} \quad \text { and } \quad \varepsilon \equiv-1 \bmod \mathfrak{P}_{2} .
$$

Proof. For brevity, put $X=\left(\mathcal{O}_{K} / \pi\right)^{\times} /\left[\mathcal{O}_{K}^{\times}\right]_{\pi}$. We have

$$
\left(\mathcal{O}_{K} / \pi\right)^{\times}=\left(\mathcal{O}_{K} / \mathfrak{P}_{1}\right)^{\times} \oplus\left(\mathcal{O}_{K} / \mathfrak{P}_{2}\right)^{\times}=\mathbb{F}_{p}^{\times} \oplus \mathbb{F}_{p}^{\times} .
$$

The Galois group $G=\operatorname{Gal}(K / F)$ fixes the prime ideal $\mathfrak{P}_{i}$, and it acts trivially on $\left(\mathcal{O}_{K} / \mathfrak{P}_{i}\right)^{\times}$. The augmentation $\iota_{G}: \mathbb{Z} G \rightarrow \mathbb{Z}$ maps both $\mathfrak{n}_{G}$ and $\theta_{G, 2}$ to $(p-1) / 2$. Hence, we see from (2) that $\iota_{G}$ maps the ideal $\mathcal{S}_{G} \subseteq \mathbb{Z} G$ onto the ideal of $\mathbb{Z}$ generated by $(p-1) / 2$. Therefore, the condition $X^{\mathcal{S}_{G}}=\{0\}$ is equivalent to

$$
\left(\mathcal{O}_{K} / \pi\right)^{\times(p-1) / 2} \subseteq\left[\mathcal{O}_{K}^{\times}\right]_{\pi}
$$

From this, we obtain the assertion.

Lemma 10. Let $F=\mathbb{Q}(\sqrt{-d})$ be an imaginary quadratic field with a square free positive integer $d$, and let $p$ be a prime number splitting in $F$. There exists a unit $\varepsilon \in \mathcal{O}_{K}^{\times}$satisfying (7) in the following two cases:

(I) $d=1$,

(II) $d$ is a prime number with $d \not \equiv 1 \bmod 4$, and $p \equiv 3 \bmod 4$.

Proof. We first show the assertion in case (II). Let $E=F(\sqrt{-p})$. It is well known that the unit index $Q_{E}$ of the imaginary abelian field $E$ equals 2 by Hasse $\left[7\right.$, p. 76]. We apply the classical argument used to show $Q_{E}=2$. Let $E^{+}=\mathbb{Q}(\sqrt{p d})$ be the maximal real subfield of $E$. Let $\mathfrak{Q}_{d}$ be the prime ideal of $E^{+}$over the prime $d ;(d)=\mathfrak{Q}_{d}^{2}$. From the conditions on $d$ and $p$, we see that the class number of $E^{+}$is odd by genus theory. Hence, there exist $u, v \in \mathbb{Z}$ such that $u^{2}-v^{2} p d= \pm 4 d$. It follows that $u=u^{\prime} d$ for some $u^{\prime} \in \mathbb{Z}$ and $\eta=\left(u^{\prime} \sqrt{-d}+v \sqrt{-p}\right) / 2$ is a unit of $\mathcal{O}_{E}$. Let $\mathfrak{P}_{1}$ and $\mathfrak{P}_{2}$ be the prime ideals of $K$ over $p$. Let $a \in \mathbb{Z}$ be an integer such that $\sqrt{-d} \equiv a \bmod \mathfrak{P}_{1}$. We see that $\sqrt{-d} \equiv-a \bmod \mathfrak{P}_{2}$ by taking the conjugate over $\mathbb{Q}$. Therefore, $\eta \equiv b \bmod \mathfrak{P}_{1}$ and $\eta \equiv-b \bmod \mathfrak{P}_{2}$ for some integer $b$ with $1 \leq b \leq p-1$. Let $\delta_{b}=1+\zeta_{p}+\cdots+\zeta_{p}^{b-1}$ be a cyclotomic unit in $K$. Then, since $\delta_{b} \equiv b \bmod \pi$, the unit $\varepsilon=\eta / \delta_{b}$ satisfies (7). 
In case (I), we can similarly show the assertion by taking $\varepsilon=\sqrt{-1}$ times a suitable cyclotomic unit of $K$.

Proof of Theorem 1. By Lemma 6, we do not need the conditional result of Weinberger [27] mentioned before. The imaginary quadratic fields $F$ with $h_{F}=1$ were determined by Stark [24]. Those with $h_{F}=2$ were determined independently by Stark [25] and Montgomery and Weinberger [23], and those with $h_{F}=4$ by Arno [1]. By genus theory, we can easily pick out those with $C l_{F}=(\mathbb{Z} / 2)^{\oplus 2}$ from Arno's result. Using these results and Lemmas 1 and 2, we obtain the following lists.

Lemma 11. An imaginary quadratic field $F=\mathbb{Q}(\sqrt{-d})$ may satisfy $\left(A_{5}\right)$ only when $d$ is one of the following:

$$
\text { (i) } 1,3,11,19 \text {; (ii) } 6,51,91 \text {; (iii) } 21 \text {. }
$$

Lemma 12. An imaginary quadratic field $F=\mathbb{Q}(\sqrt{-d})$ may satisfy $\left(A_{7}\right)$ only when $d$ is one of the following:
(i) 3,19 ;
(ii) $5,6,10,13,115,187$;
(iii) 33,195 .

Lemma 13. An imaginary quadratic field $F=\mathbb{Q}(\sqrt{-d})$ may satisfy $\left(A_{11}\right)$ only when $d$ is one of the following:
(i) $2,7,19,43$;
(ii) $6,10,13,35,51,123,403$;

(iii) $21,30,57,85,195,435,483$.

In the above lists, those $F$ or $d$ in the first groups satisfy $h_{F}=1$, those in the second groups have $h_{F}=2$, and those in the last groups, $C l_{F}=(\mathbb{Z} / 2)^{\oplus 2}$. In the following, let $K=F\left(\zeta_{p}\right)$ and $E$ be the intermediate field of $K / F$ with $[E: F]=2$. Let $\varrho$ be a generator of $G=\operatorname{Gal}(K / F)$. By $(2), \mathcal{S}_{G}$ is generated by

$$
\mathfrak{n}_{G}=1+\varrho+\cdots+\varrho^{(p-1) / 2-1} .
$$

All the following calculations were done using KASH.

The case $p=5$. We checked that the natural map $C l_{F} \rightarrow C l_{E}$ is not trivial when $d=6,51,91$ or 21 . Hence, by Lemma $4, F$ does not satisfy $\left(A_{5}\right)$ for these $d$. When $d=1$ or 3 , we have $C l_{K}=\{0\}$. When $d=1$, we see that $C l_{K}(\pi)^{\mathcal{S}_{G}}=\{0\}$ by Lemmas 9 and 10 . When $d=3$, we checked $C l_{K}(\pi)^{\mathcal{S}_{G}}=\{0\}$ by explicitly finding a system of fundamental units of $K$. Hence, by Proposition $5, F$ satisfies $\left(A_{5}\right)$ for $d=1$ or 3 . When $d=11$ (resp. 19), we see that $C l_{K}=\mathbb{Z} / 2$ (resp. $\mathbb{Z} / 4$ ) and $C l_{K}^{\mathcal{S}_{G}}=\{0\}$. We chose an ideal $\mathfrak{A}$ of $K$ such that the class $[\mathfrak{A}]$ generates the cyclic group $C l_{K}$. We checked that a generator $\alpha$ of the principal ideal $\mathfrak{A}^{1+\varrho}$ is not congruent to a unit modulo $\pi$. Hence, by Proposition $5, F$ does not satisfy $\left(A_{5}\right)$ for $d=11$ or 19 . 
The case $p=7$. We checked that the natural map $C l_{F} \rightarrow C l_{E}$ is not trivial when $d=6,33,195$. Hence, by Lemma $5, F$ does not satisfy $\left(A_{7}\right)$ for these $d$. For $d=5,10,115,187$, the 2-part of $C l_{K}$ is nontrivial and cyclic, and hence $F$ does not satisfy $\left(A_{7}\right)$ by Lemma 7 . When $d=13$, we found that $C l_{K}=\mathbb{Z} / 2^{\oplus 3} \oplus \mathbb{Z} / 3$ and $C l_{K}^{\mathcal{S}_{G}} \neq\{0\}$, and hence $F$ does not satisfy $\left(A_{7}\right)$. When $d=19$, we found that $C l_{K}=\mathbb{Z} / 3$ and $C l_{K}^{\mathcal{S}_{G}}=\{0\}$. We checked that $F$ does not satisfy $\left(A_{7}\right)$ in this case similarly to the case where $p=5$ and $d=11,19$. Finally, when $d=3$, we found that $C l_{K}=\{0\}$, and that $C l_{K}(\pi)^{\mathcal{S}_{G}}=\{0\}$ by Lemmas 9 and 10. Hence, $F$ satisfies $\left(A_{7}\right)$ for $d=3$.

The case $p=11$. For $d=10,35,21,30,57,85,195,435$ or 483 , we found that the natural map $C l_{F} \rightarrow C l_{E}$ is not trivial. Hence, by Lemma $5, F$ does not satisfy $\left(A_{11}\right)$ for these $d$. For $d=6,13,51,123$ or 403 , we have $h_{E}=2$. Hence, by Lemma $7, F$ does not satisfy $\left(A_{11}\right)$ for these $d$. For $d=43$, we have $h_{E}=3$, and $F$ does not satisfy $\left(A_{11}\right)$ by Lemma 8 . Let us deal with the remaining cases where $d=2,7$ or 19 . In these cases, we have $h_{E}=1$. Instead of the field $K=F\left(\zeta_{11}\right)$, we use the subfield $N=F(\cos 2 \pi / 11)$. We have $h_{N}=5$ for $d=2$ or 7 , and $h_{N}=55$ for $d=19$. Let $\mathfrak{A}$ be an ideal of $N$. If $F$ satisfies $\left(A_{11}\right)$, then $\mathfrak{A}^{\mathfrak{n}_{G}} \mathcal{O}_{K}=\alpha \mathcal{O}_{K}$ for some $\alpha \in K^{\times}$congruent modulo $\pi$ to a unit of $K$. Taking the norm to $N$, it follows that $\mathfrak{A}^{2 \mathfrak{n}_{G}}=\beta \mathcal{O}_{N}$. Here, $\beta=N_{K / N} \alpha$ and is congruent to a unit of $N$ modulo $\pi$. For these three $d$, we chose a nontrivial ideal $\mathfrak{A}$ of $N$ and checked that $\mathfrak{A}^{2 \mathfrak{n}_{G}}$ is a principal ideal of $\mathcal{O}_{N}$ and that its generator is not congruent to a unit of $N$ modulo $\pi$ after computing a system of fundamental units of $N$. Therefore, there exists no imaginary quadratic field satisfying $\left(A_{11}\right)$.

OBSERVATION/Question. Let $p$ be a prime number. As usual, we put $\widetilde{p}=4($ resp. $p)$ when $p=2$ (resp. $p \geq 3)$. We have seen that for the first five $\widetilde{p}$, the number of imaginary quadratic fields $F$ satisfying $\left(A_{p}\right)$ is $4,3,2,1$ and 0 , respectively. What is the next term or a general term of this (arithmetic!) progression?

REMARK 1. We can generalize Lemma 3 as follows. For a number field $F$, let $\mu_{F}$ be the group of roots of unity in $F$, and $\mu_{F}^{1}$ the subgroup of elements of odd order. Let $K / F$ be a finite cyclic extension with both $K$ and $F$ CM-fields. Assume that the following three conditions are satisfied:

(i) $2^{e} \|[K: F]$ for some $e \geq 1$,

(ii) $\mu_{F}=\left\langle\zeta_{2}{ }^{e}\right\rangle$,

(iii) there exists a prime ideal $\wp$ of $F$ over an odd prime number $p$ such that $\wp$ is totally ramified at the intermediate field $E$ of $K / F$ with $[E: F]=2^{e}$.

By the last condition, we can write $E=F\left(a^{1 / 2^{e}}\right)$ for some $a \in F^{\times}$with $\operatorname{ord}_{\wp}(a)=1$. Then we can show that the exponent of the kernel of the 
natural map $C l_{F}^{-} \rightarrow C l_{K}^{-}$divides 2 by an argument exactly similar to the proof of Lemma 3 using $\mu_{K}^{1}$ and $a^{1 / 2^{e}}$ in place of $\mu_{p}$ and $\sqrt{p^{*}}$.

REMARK 2. If all imaginary abelian fields $K$ of degree $2(p-1)$ for which $C l_{K}^{2 h_{p}^{-}}=\{0\}$ were determined, it would be possible to determine all real quadratic fields satisfying $\left(A_{p}\right)$ for small primes $p$ by Proposition 6 .

4. Subfields of the $p$-cyclotomic field. In this section, we deal with subfields of the $p$-cyclotomic field $\mathbb{Q}\left(\zeta_{p}\right)$. The following is an immediate consequence of Proposition 1. A more general statement is given in [9, Proposition 3.4].

Proposition 8. Let $p$ be an odd prime number. An imaginary subfield $F$ of $\mathbb{Q}\left(\zeta_{p}\right)$ satisfies $\left(A_{p}\right)$ if and only if $p=3$ and $F=\mathbb{Q}\left(\zeta_{3}\right)$.

In the following, we summarize what is known or conjectured for the real case. Let $\mathcal{O}_{F}^{\prime}=\mathcal{O}_{F}[1 / p]$ be the ring of $p$-integers of $F$. We say that $F$ satisfies condition $\left(A_{p}^{\prime}\right)$ if for any $\Gamma$-extension $N / F, \mathcal{O}_{N}^{\prime}$ is cyclic over the group ring $\mathcal{O}_{F}^{\prime} \Gamma$. It is known that if $F$ satisfies $\left(A_{p}\right)$ then it satisfies $\left(A_{p}^{\prime}\right)$. Condition $\left(A_{p}^{\prime}\right)$ is easier to handle than $\left(A_{p}\right)$, and many results on $\left(A_{p}^{\prime}\right)$ are already obtained in $[14,16,17,18]$. Let $K=F\left(\zeta_{p}\right)$. For instance, it is known that $F$ satisfies $\left(A_{p}^{\prime}\right)$ if $h_{K}^{\prime}=1$, where $h_{K}^{\prime}$ is the class number of the Dedekind domain $\mathcal{O}_{K}^{\prime}$.

Let $K=\mathbb{Q}\left(\zeta_{p}\right)$, and $h_{p}$ the class number of $K$. As the unique prime ideal of $\mathcal{O}_{K}$ over $p$ is principal, we have $h_{p}=h_{K}^{\prime}$. It is well known that $h_{p}=1$ if and only if $p \leq 19$ (cf. [26, Theorem 11.1]). Hence, when $p \leq 19$, any subfield $F$ of $K=\mathbb{Q}\left(\zeta_{p}\right)$ satisfies $\left(A_{p}^{\prime}\right)$. When $p \geq 23$, we proposed the following conjecture in [18].

Conjecture 1 . Let $p$ be a prime number with $p \geq 23$, and $F$ a subfield of $\mathbb{Q}\left(\zeta_{p}\right)$ with $F \neq \mathbb{Q}$. If $[F: \mathbb{Q}]>2$ or $p \equiv 1 \bmod 4$, then $F$ does not satisfy condition $\left(A_{p}^{\prime}\right)$ except when $p=29$ and $[F: \mathbb{Q}]=2$ or 7 .

We have seen in [18, Proposition 4] that the conjecture is valid when $23 \leq p \leq 499$ and when $[K: F] \leq 4$ or $=6$. A reason that the case $p=29$ is exceptional is that $h_{p}^{-}$is a power of 2 if and only if $p \leq 19$ or $p=29$ by Horie $[10]$. When $p=29$ and $[F: \mathbb{Q}]=2$ or 7 , it is known that $F$ satisfies $\left(A_{p}^{\prime}\right)([18$, Proposition $4(\mathrm{II})])$. In [16, Theorem 1], we determined all imaginary subfields $F$ of $\mathbb{Q}\left(\zeta_{p}\right)$ satisfying $\left(A_{p}^{\prime}\right)$, and gave an affirmative answer to the conjecture for the imaginary case. In [17], we showed the following assertion for the real case.

Proposition 9 ([17, Proposition 1]). Let $p \geq 23$. Assume that $q \| h_{p}^{-}$ for some odd prime number $q$. Then any real subfield $F$ of $\mathbb{Q}\left(\zeta_{p}\right)$ with $F \neq \mathbb{Q}$ does not satisfy $\left(A_{p}^{\prime}\right)$. (Hence, it does not satisfy $\left(A_{p}\right)$.) 
The assumption in this assertion is satisfied for all primes $p$ with $23 \leq$ $p<2^{10}$ except $p=29,31,41$ by the tables in [26], Lehmer and Masley [19] and Yamamura [28].

Now, we have enough reasons to propose the following:

Conjecture 2. A real subfield $F$ of $\mathbb{Q}\left(\zeta_{p}\right)$ with $F \neq \mathbb{Q}$ does not satisfy $\left(A_{p}\right)$ except when $p \leq 19$, or $p=29$ and $[F: \mathbb{Q}]=2,7$.

Among the exceptional cases in Conjecture 2, we have checked that $\mathbb{Q}(\sqrt{5})$ satisfies $\left(A_{5}\right)$ and that $\mathbb{Q}(\cos 2 \pi / 7)$ does not satisfy $\left(A_{7}\right)$ by a computer calculation based upon Theorem 2. The difficult point is that the locally free class group $C l^{0}\left(\mathcal{O}_{F} \Gamma\right)$ is very complicated when $F / \mathbb{Q}$ is ramified at $p$.

Acknowledgements. The authors are grateful to the referee for carefully reading the original manuscript and for several kind suggestions. Thanks to the suggestions, we added Remark 1 and considerably improved the proof of Lemma 6 . The first author was partially supported by Grantin-Aid for Scientific Research (C), (No. 16540033), the Ministry of Education, Culture, Sports, Science and Technology of Japan. The second author was partially supported by Grant-in-Aid for Young Scientists (No. 16740019), the Ministry of Education, Culture, Sports, Science and Technology of Japan.

\section{References}

[1] S. Arno, The imaginary quadratic fields of class number 4, Acta Arith. 60 (1992), 321-334.

[2] J. Brinkhuis, Normal integral bases and complex conjugation, J. Reine Angew. Math. 375/376 (1987), 157-166.

[3] J. E. Carter, Normal integral bases in quadratic and cyclic cubic extensions of quadratic fields, Arch. Math. (Basel) 81 (2003), 266-271; Erratum, ibid. 83 (2004), no. 6 , vi-vii.

[4] M. Conrad and D. R. Replogle, Nontrivial Galois module structure of cyclotomic fields, Math. Comp. 72 (2003), 891-899.

[5] E. J. Gómez Ayala, Bases normales d'entiers dans les extensions de Kummer de degré premier, J. Théor. Nombres Bordeaux 6 (1994), 95-116.

[6] C. Greither, D. R. Replogle, K. Rubin and A. Srivastav, Swan modules and HilbertSpeiser number fields, J. Number Theory 79 (1999), 164-173.

[7] H. Hasse, Über die Klassenzahl Abelscher Zahlkörper, Akademie-Verlag, Berlin, 1952.

[8] E. Hecke, Lectures on the Theory of Algebraic Numbers, Springer, Berlin, 1981.

[9] T. Herreng, Sur les corps de Hilbert-Speiser, J. Théor. Nombres Bordeaux 17 (2005), $767-778$.

[10] K. Horie, On the class numbers of cyclotomic fields, Manuscripta Math. 65 (1989), 465-477. 
[11] H. Ichimura, Note on the ring of integers of a Kummer extension of prime degree, $V$, Proc. Japan Acad. 78A (2002), 76-79.

[12] -, Normal integral bases and ray class groups, Acta Arith. 114 (2004), 71-85.

[13] —, Normal integral bases and ray class groups, II, Yokohama Math. J. 53 (2006), $75-81$.

[14] -, Stickelberger ideals and normal bases of rings of p-integers, Math. J. Okayama Univ. 48 (2006), 9-20.

[15] - A class number formula for the p-cyclotomic field, Arch. Math. (Basel) 87 (2006), $539-545$.

[16] - , Hilbert-Speiser number fields for a prime $p$ inside the p-cyclotomic field, submitted for publication.

[17] —, Triviality of Stickelberger ideals of conductor $p$, J. Math. Sci. Univ. Tokyo 13 (2006), 617-628.

[18] H. Ichimura and H. Sumida-Takahashi, Stickelberger ideals of conductor $p$ and their application, J. Math. Soc. Japan 58 (2006), 885-902.

[19] D. H. Lehmer and J. Masley, Table of the cyclotomic class numbers $h^{*}(p)$ and their factors for $200<p<521$, Math. Comp. 32 (1978), 577-582.

[20] L. R. McCulloh, A Stickelberger condition on Galois module structure for Kummer extensions of prime degree, in: Algebraic Number Fields, L-Functions and Galois Properties (Durham, 1975), A. Fröhlich (ed.), Academic Press, London, 1977, 561-588.

[21] —, Galois module structure of elementary abelian extensions, J. Algebra 82 (1983), 102-134.

[22] I. Miyada, On imaginary abelian number fields of type $(2, \ldots, 2)$ with one class in each genus, Manuscripta Math. 88 (1995), 535-540.

[23] H. L. Montgomery and P. J. Weinberger, Notes on small class numbers, Acta Arith. 24 (1974), 529-542.

[24] H. M. Stark, A complete determination of the complex quadratic fields of classnumber one, Michigan Math. J. 14 (1967), 1-27.

[25] - On complex quadratic fields of class-number two, Math. Comp. 29 (1975), 289-302.

[26] L. C. Washington, Introduction to Cyclotomic Fields, 2nd ed., Springer, Berlin, 1997.

[27] P. J. Weinberger, Exponents of the class groups of complex quadratic fields, Acta Arith. 22 (1973), 117-124.

[28] K. Yamamura, Table of relative class numbers of imaginary abelian fields of prime power conductor $<2^{10}=1024$, ftp://tnt.math.metro-u.ac.jp/pub/table/rcn/.

Faculty of Science

Ibaraki University

Bunkyo 2-1-1, Mito

Ibaraki, 310-8512, Japan

E-mail: hichimur@mx.ibaraki.ac.jp
Faculty and School of Engineering The University of Tokushima 2-1, Minami-josanjima-cho

Tokushima, 770-8506, Japan E-mail: hiroki@pm.tokushima-u.ac.jp

Received on 2.8.2006

and in revised form on 1.11.2006 